\title{
Gamma Radiation Effects on Siloxane-Based Additive Manufactured Structures
}

Andrew M. Schmalzer, Carl M. Cady, Drew Geller, Denisse Ortiz-Acosta, Adam T. Zocco, Jamie Stull and Andrea Labouriau*

Los Alamos National Laboratory, Los Alamos, NM 87545

\begin{abstract}
Siloxane-based additive manufactured structures prepared by the direct ink write (DIW) technology were exposed to ionizing irradiation in order to gauge radiolysis effects on structure-property relationships. These well-defined 3-D structures were subjected to moderate doses of gamma irradiation in an inert atmosphere and characterized by a suite of experimental methods. Changes in thermal, chemical, microstructure, and mechanical properties were evaluated by DSC, TGA, FT-IR, mass spectroscopy, EPR, solvent swelling, SEM, and uniaxial compressive load techniques. Our results demonstrated that 3-D structures made from aromatic-free siloxane resins exhibited hardening after being exposed to gamma radiation. This effect was accompanied by gas evolution, decreasing in crystallization levels, decreasing in solvent swelling and damage to the microstructure. Furthermore, long-lived radiation-induced radicals were not detected by EPR methods. Our results are consistent with cross-link formation being the dominant degradation mechanism over chain scission reactions. On the other hand, 3-D structures made from high phenyl content siloxane resins showed little radiation damage as evidenced by low off gassing.
\end{abstract}

*Corresponding author, email: andrea@lanl.gov , phone: 505-667-7216

Keywords: radiolysis, DIW resin, additive manufacturing, polysiloxanes. 


\section{Introduction.}

Additive manufacturing (AM) technology allows for the production of complex materials with customized design and improved performance, along with reducing manufacturing time, costs, and the amounts of feedstock and waste. ${ }^{1}$ Recently, such technology gained considerable attention since it enabled the design of new and exciting devices, ranging from self-healing materials to artificial organs. ${ }^{2,3}$ A commercially available siloxane-based adhesive known as Dow Corning ${ }^{\circledR}$ SE1700, was identified by Duoss and co-workers as a suitable polymeric resin to be used in the direct ink writing (DIW) process (Figure 1). ${ }^{4}$ Their elegant DIW approach produced well-defined 3-D porous elastomeric architectures that exhibited directional load response together with negative stiffness. The authors demonstrated that the mechanical response of these 3-D structures was a function of the micro-architected design, which for instance, could be tailored in interesting ways to prevent damage to the material when under stress.
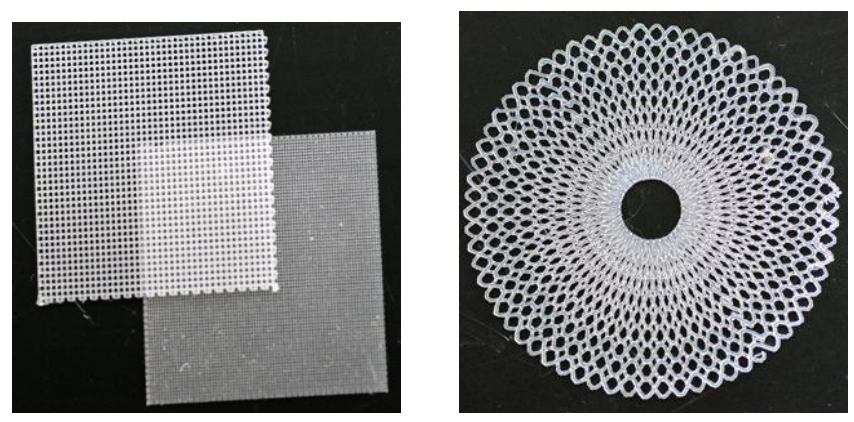

Figure 1. Examples of AM pads produced using the SE1700 resin.

These novel AM siloxane pads may be used in applications that require exposure to harsh environmental conditions such as ionizing radiation. Thus, the present work investigated the resilience of such AM structures when exposed to relatively moderate doses of gamma radiation, namely less than $250 \mathrm{kGy}$. As mentioned above, the DIW resin of interest is based on poly(dimethyl siloxane) (PDMS), a polymer that is non-toxic and well-known for its thermal and chemical stability. Exposure of PDMS to high energy irradiation such as gamma rays has been the subject of several investigations over the years, showing that the polymer is susceptible to 
radiation-induced structural changes. For instance, Charlesby and others described formation of a cross-linked network accompanied by gas evolution. ${ }^{5-7}$ Radiation-induced changes in crosslink density are expected to influence the material's mechanical response by enhancing tensile properties and increasing hardness, as it was certainly observed by Warrick and Basfar. ${ }^{8,9}$

To our knowledge, the current work represents the first study on radiolysis of 3-D structures produced by the DIW technique. Radiation-induced changes in chemistry, thermal properties, microstructure, and mechanical response were assessed by a combination of diverse experimental techniques, providing valuable insights on the radiolysis in inert atmospheres of AM siloxane pads.

\section{Materials and Characterization Methods.}

\subsection{Materials.}

One of the materials of interest to this study is a commercial two-part silicone adhesive, known as Dow Corning ${ }^{\circledR}$ SE 1700 . Preparation of the resin requires mixing "Part A" and "Part B" in a 10 to 1 ratio, respectively. This is a non-flowing (yield stress) resin that cures after 30 minutes at $150^{\circ} \mathrm{C}$, allowing for good working time after the mixing. This curing procedure was investigated by varying the curing time from 30 minutes at $150^{\circ} \mathrm{C}$ to up to 48 hours at $150^{\circ} \mathrm{C}$. TGA results showed that the weight loss was mostly independent of the curing time, indicating that curing for 30 minutes is largely sufficient. According to the material data sheet provided by the vendor, part $A$ is composed, by weight, of $55-75 \%$ dimethylsiloxane (dimethylvinylterminated), 15-35\% trimethylated silica, 5-10\% dimethyl vinylated and trimethylated silica, 1$5 \%$ titanium oxide, and less than $1 \%$ methylvinyl, (dimethoxy (glycidoxypropyl)-terminated). Part $\mathrm{B}$ is composed of $30-50 \%$ dimethylsiloxane (dimethylvinyl-terminated), $30-50 \%$ dimethyl methylhydrogen siloxane, and $15-35 \%$ dimethyl vinylated and trimethylated silica. ${ }^{1} \mathrm{H}$ NMR analysis of the uncured resin confirms that it contains vinyl and silane groups. Analysis by inductively coupled plasma mass spectrometry (ICP-MS) reveals that several metals are also found in small quantities in parts A and B, as listed in Table 1. The platinum detected in part A is assumed to be the catalyzing agent for the curing process of the SE 1700 resin. 
Table 1. ICP-MS data obtained for parts A and B (in ppm). Other metals that were detected in the resin included osmium, nickel and gold in part A, and silver in both parts.

\begin{tabular}{|l|c|c|c|c|c|}
\hline & Sn & Ca & Ti & Cu & Pt \\
\hline Part A & 2.83 & 24.16 & 2.4 & 0.54 & 7.2 \\
\hline Part B & 4.70 & 0 & 1.45 & 0.32 & 0 \\
\hline
\end{tabular}

Another material of interest to this work is a commercially available Dow Corning ${ }^{\circledR}$ siloxane resin known as OE6636. This is a two part optical encapsulant with high diphenyl content and high viscosity (7500 cP). The uncured resin was modified to prevent the material from flowing under its own weight during 3-D printing. Untreated fumed silica (Cab-o-sil ${ }^{\circledR}$ HS-5) was added to uncured OE6636 using a Thinky planetary mixer. The addition of 8.5 wt\% silica made this material printable through a $600 \mu \mathrm{m}$ nozzle. The 3-D printed pad was cured following the same procedure as for the SE1700 resin.

AM pads were produced using the DIW process, which differs from traditional 3D printing methods by using a thermosetting shear-thinning yield-stress resin that holds its shape during the printing and curing process. This technique has been recently demonstrated to manufacture structure-controlled materials. ${ }^{4}$ DIW allows for microstructural control of the final material, as opposed to blown closed cell foams that have stochastic cellular structures. The AM pads investigated in this study were printed using a Nordson Ultimus $V$ engineered fluid dispenser (EFD) and Aerotech 3 axis linear positioning stage. The EFD provides a constant pressure drop to a syringe filled with uncured SE1700, which is mounted on the z-axis of the Aerotech stage. The $x, y$, and $z$ axes are controlled by the Aerotech Npaq, and operated according to a gcode based program written in the Aerotech A3200 Motion Composer Software.

Once the printed material was cured, approximately $21 \mathrm{~g}$ samples were cut from the full pad, and placed in aluminum canisters. The canisters were sealed with copper gasket, evacuated to $10^{-3}$ torr and then backfilled with 650 torr of high purity nitrogen gas. These canisters were exposed to cobalt-60 sources at a constant dose rate of $1 \mathrm{~Gy} / \mathrm{sec}$. The samples were irradiated for different time periods to achieve total doses of 10,50, 100 and $200 \mathrm{kGy}$. These exposures 
were performed at the Gamma Irradiation Facility (GIF) at Sandia National Laboratories in New Mexico.

2.2. Thermal Analyses. TGA experiments were carried out using a commercial TA Instruments Q5000. 10 to $20 \mathrm{mg}$ samples were heated from $30^{\circ} \mathrm{C}$ to $750^{\circ} \mathrm{C}$ at $2{ }^{\circ} \mathrm{C} / \mathrm{min}$ under nitrogen atmosphere. DSC experiments were performed on a TA Instruments Q2000 DSC with a liquid nitrogen cooling (LNC) accessory. Approximately 5 to $10 \mathrm{mg}$ sample were sealed in a Tzero aluminum hermetic pan. The cooling-heating cycle consisted of equilibrating the sample to $35{ }^{\circ} \mathrm{C}$, cool down to $-20^{\circ} \mathrm{C}$ at $10{ }^{\circ} \mathrm{C} / \mathrm{min}$, cool down to $-150{ }^{\circ} \mathrm{C}$ at $5^{\circ} \mathrm{C} / \mathrm{min}$, isotherm at $-150{ }^{\circ} \mathrm{C}$ for 15 minutes, heat up to $100^{\circ} \mathrm{C}$ at $10^{\circ} \mathrm{C} / \mathrm{min}$. Both the temperature and heat of fusion were calibrated using the melting of high purity Indium. The pristine polymer was characterized by a melting temperature ca. $-44.5^{\circ} \mathrm{C}$ and a glass transition temperature at about $-120{ }^{\circ} \mathrm{C}$, which are typical for siloxanes.

2.3. FTIR. The IR absorption spectra were obtained with a Nicolet Avatar 360 benchtop FTIR operating in Attenuated Total Reflectance (ATR) mode. Data was collected using a Smart DuraSampleIR ATR accessory equipped with a diamond crystal. The IR detector was deuterated tri-glycine sulfate (DTGS). All data was taken with a resolution of $8 \mathrm{~cm}^{-1}$, and represent the average of 32 scans.

2.4. Mass spectroscopy. The headspace of each canister containing the irradiated AM pads was analyzed by mass spectroscopy using a Finnigan 271 mass spectrometer. Gas samples were collected into small gas bottles using a small vacuum manifold. An aliquot of a sample gas was injected to the mass spectrometer inlet chamber. From the inlet chamber, the gas was allowed to leak slowly into the mass spectrometer ionizer during an analysis. Initially, qualitative spectral scans were performed for each unknown gas sample from mass 45 up to mass $390+$, and a selection of peaks to be scanned quantitatively was assembled. Detectors were calibrated by known mixtures of gases, wherein detector sensitivities to the ions of specific gases were determined. When known mixtures or pure gases were not available for calibration, sensitivity estimates were performed. The partial pressures of inlet gases that produced the observed ion data were deduced from detector sensitivities. The sum of the molecular gas partial pressures was ratioed to the total measured sample inlet pressure, and the result was 
close to $100 \%$ for acceptable analyses. The NIST mass spectral database was consulted for assignment of hydrocarbons in the headspace analyses.

2.5. Solvent Swelling. These experiments were performed to estimate changes in cross-link density as a result of gamma exposure. Four specimens for each condition were analyzed by this technique. The samples $(0.12 \mathrm{~g})$ were first weighed for the initial dry weight and then immersed in toluene (Fisher Scientific, 99\% purity). The swollen samples were periodically weighed until reaching saturation and the equilibrium weights obtained in toluene were recorded at the end of the experiment.

2.6. SEM imaging. An FEI Inspect $F$ field emission SEM instrument was used to analyze the microstructure of the AM materials. Surface analysis was carried out on $1 \mathrm{~cm}^{2}$ samples, and placing them onto standard mounts with carbon tape facing up. To avoid charging effects, the edges of the samples were painted with graphite paint and then sputter coated with about 5 $\mathrm{nm}$ of gold-palladium.

2.7. EPR measurements. Experiments at X-band were performed on a Bruker model E500 (Bruker BioSpin, Billerica, MA) equipped with a Bruker SHQE resonator. CW spectra were collected at room temperature. Spectra were acquired using a modulation amplitude of $10 \mathrm{G}, \mathrm{a}$ frequency modulation of $100 \mathrm{kHz}$, a microwave power of $5 \mathrm{~mW}, 40$ scans per sample and a receiver gain of $60 \mathrm{~dB}$. CW EPR spectroscopy was performed on samples of AM pad that had been exposed to varying levels of gamma irradiation and a control sample.

2.8. Mechanical testing. Each of the irradiated AM pads was subjected to cyclic compression test in order to identify how each level of dose affected the mechanical properties. Sample dimensions were approximately $7 \mathrm{~mm}$ by $7 \mathrm{~mm}$ long and $3 \mathrm{~mm}$ tall, and the average density was $0.61 \mathrm{~g} / \mathrm{cc}$. The samples were tested at room temperature and at $-60{ }^{\circ} \mathrm{C}$ at a strain rate of $0.01 / \mathrm{s}$ to $\sim 60 \%$ engineering strain. The compression tests were conducted with an MTS model 880 test frame with a second 100lb load cell and an extensometer to control and measure displacement more accurately. The extensometer used adds a small additional load due to the spring constant of the device but it can be measured and subtracted from the experimental data if it is a high enough percentage of the overall load signal. Generally the load increase is about $0.75 \mathrm{~N} / 0.5 \mathrm{~mm}$ of displacement, and it is linear over its range of accuracy $\left(0.5^{\prime \prime}\right.$ 
displacement range). This bias load was determined to be less than $3 \%$ of sample load for the AM pads. The analysis of the current results does not subtract away this known bias error since this error is consistent for all tests. The benefit to using this extensometer is that it eliminates system compliance and hysteresis that can be seen in the LDVT signal, thus reducing the uncertainty of the load/displacement for the entire system. There was a variation of about $2{ }^{\circ} \mathrm{C}$ during the testing, and the duration of each test was approximately 68 minutes. The experiments were run by placing the samples on optically flat tungsten carbide platens, centered in the loading system, with no lubrication. The tests were run by finding the contact of the plattens and setting that as zero displacement, opening to a gap large enough to insert the samples, bring them into near contact with the sample to increase the thermal conductivity into the material. Then run to a gap that is approximately $60 \%$ engineering strain. This enables one to determine contact during each test, make sure the sample is fully unloaded between cycles and ensure no pre-strain is put on the sample during set up.

\section{Results and Discussion.}

\subsection{Irradiation Effects on Chemical Properties.}

Gas analysis of the headspace provides useful insights on polymer degradation mechanisms since gases are formed due to atom or side-chain abstraction, and are then indicative of damage to the polymer. The radiation chemistry of PDMS has been extensively investigated over the years showing that hydrogen, methane and ethane are the major volatile products. $^{5}$ Gas evolution is accompanied by cross-link formation as first proposed by Charlesby. ${ }^{10}$ Initially, this degradation mechanism was considered to be the main radiolysis mechanism, occurring as a result of $\mathrm{C}-\mathrm{H}$ and $\mathrm{C}$-Si bond scission. The $\mathrm{G}(\mathrm{X})$-value in PDMS, which represents the number of cross-links generated per $100 \mathrm{eV}$ of energy absorbed (1/100 eV is equivalent to $1.036 \mathrm{e}-7 \mathrm{~mol} / \mathrm{J}$ ); was found to be independent of the polymer molecular weight and equal to 3. Other studies have shown large variations in the yield of the volatiles: $G\left(H_{2}\right)=$ 0.3 to $1.25, \mathrm{G}\left(\mathrm{CH}_{4}\right)=1.0$ to $1.3, \mathrm{G}\left(\mathrm{C}_{2} \mathrm{H}_{6}\right)=0.3$ to 0.76 . $^{7,7,11}$ These differences are probably a consequence of slightly different radiation exposures as well as variations on the material itself 
like presence of impurities and additives. Along with gas analysis, infrared spectroscopy has been comprehensively used to characterize radiolysis in PDMS and has identified the nature of some of the radiation-induced cross-links. ${ }^{7,11,12}$ As an example, Miller estimated the yields for the silylmethylene cross-links ( $\equiv \mathrm{SiCH}_{2} \mathrm{Si} \equiv$ ) to be 1.8 , silylethylene cross-links ( $\left.\equiv \mathrm{SiCH}_{2} \mathrm{CH}_{2} \mathrm{Si} \equiv\right)$ to be 0.5 , and by a bromination method, Si-Si H-type cross-links were also observed with a yield value of 1.1. ${ }^{7}$ More recently, Hill and co-workers used NMR spectroscopy to identify new structural units in PDMS exposed to gamma irradiation under vacuum at $303 \mathrm{~K} \cdot{ }^{13-15}$ These new linkages are consistent with main chain scission and cross-linking formation as initially proposed by Charlesby. In addition to H-linking, Y-linking was also detected as shown in Figure 2. The author's $G$-values at various absorbed doses indicated $G(S)=1.3 \pm 0.2$ for chain scission, $G(H)=$ $0.34 \pm 0.02$ for $\mathrm{H}$-linking, and $\mathrm{G}(\mathrm{Y})=1.70 \pm 0.09$ for $\mathrm{Y}$-linking.

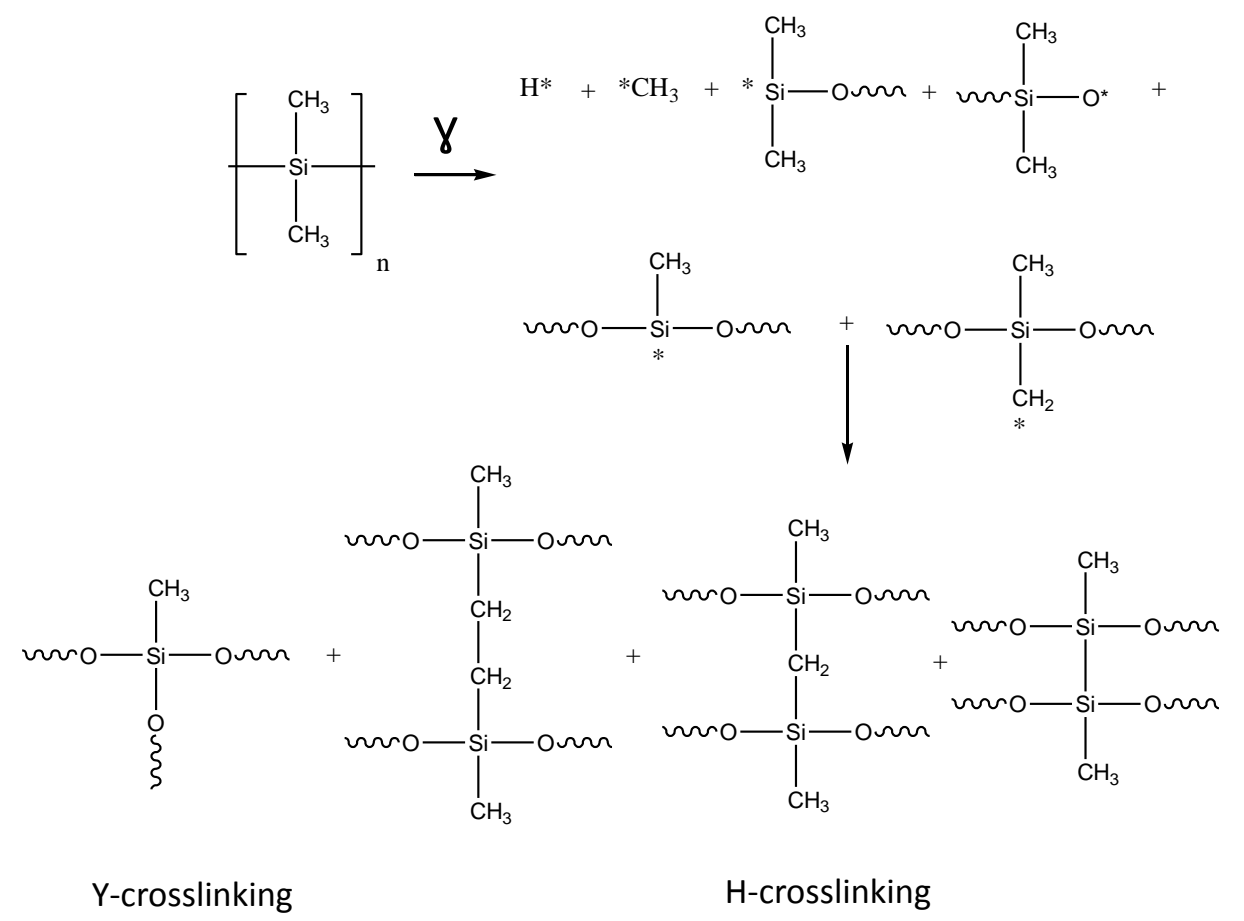

Figure 2. Scheme showing main chain scission and hydrogen and methyl abstractions from the polymer chain, which lead to gas evolution and the formation of two different types of crosslinks ( $\mathrm{Y}$ and $\mathrm{H}$ ) (reproduced from reference 26$)$. 
In the present work, mass spectroscopy was used to detect gases and volatiles evolved as a result of exposing the SE1700 AM pad to ionizing radiation. Results are listed in Table 2, and show the presence of the expected permanent gases as well as of a few other volatiles. As anticipated, the most abundant gases are hydrogen, methane, and ethane. Our results demonstrate that the concentration of these species increase approximately linearly with the gamma dose. If one assumes that the SE1700 resin contains ca. 30wt\% filler (as indicated by the manufacturer), then the estimated $G$ values for the permanent gases are $G\left(H_{2}\right)=1.6, G\left(C_{4}\right)=1.3$, and $\mathrm{G}\left(\mathrm{C}_{2} \mathrm{H}_{6}\right)=0.18$, with $\mathrm{G}$ (total) close to 3.1. Low concentrations of propane, butane and cyclic siloxanes such as hexamethylcyclotrisiloxane or D3 $\left((\mathrm{SiO})_{3}\left(\mathrm{CH}_{3}\right)_{6}\right)$ and octamethylcyclotetrasiloxane or D4 $\left(\left((\mathrm{SiO})_{4}\left(\mathrm{CH}_{3}\right)_{8}\right)\right.$ were also detected in the headspace. It is important to notice that the oxygen concentration in the headspace was less than $1 \mathrm{ppm}$, thus excluding oxidative degradation as a mechanism that could potentially contribute to the polymer degradation. ${ }^{16}$

Table 2. Concentrations in ppm of the major constituents that were detected in the headspace of irradiated SE1700 AM pad as a function of gamma exposure. Samples were exposed to gamma irradiation under $\mathrm{N}_{2}$ at room temperature and constant dose rate.

\begin{tabular}{|c|c|c|c|c|c|c|}
\hline $\begin{array}{c}\text { Gamma dose } \\
(\mathrm{kGy})\end{array}$ & $\mathrm{H}_{2}$ & $\mathrm{CH}_{4}$ & $\mathrm{C}_{2} \mathrm{H}_{6}$ & $\mathrm{C}_{3} \mathrm{H}_{8}$ & $\mathrm{C}_{4} \mathrm{H}_{10}$ & $\mathrm{Si}\left(\mathrm{CH}_{3}\right)_{4}$ \\
\hline 10 & 4,200 & 3,100 & 500 & 1.8 & 0.7 & 3.7 \\
\hline 50 & 19,500 & 14,400 & 2,300 & 13.4 & 2.9 & 17.9 \\
\hline 100 & 42,600 & 33,100 & 4,600 & 29.5 & 6.1 & 31.7 \\
\hline 200 & 71,300 & 62,900 & 8,000 & 64.4 & 13.9 & 35.3 \\
\hline
\end{tabular}

Changes in cross-link density were evaluated by solvent swelling experiments, which is one of the preferred methods to gauge variations in the network due to its easiness to perform. The irradiated AM pads were swollen in toluene until reaching saturation. Swelling results 
indicated a sharp decrease in solvent uptake with increasing dose, as shown in Figure 3. For instance, the AM pad exposed to $200 \mathrm{kGy}$ swells almost $30 \%$ less than the control one. This reduction in swelling is a direct result of an increase in cross-link density, which prevents network expansion in the solvent. Increasing in cross-ink density is expect to induce significant changes in mechanical properties, such as increase in elastic modulus and hardness accompanied by reduction in elongation. ${ }^{17}$

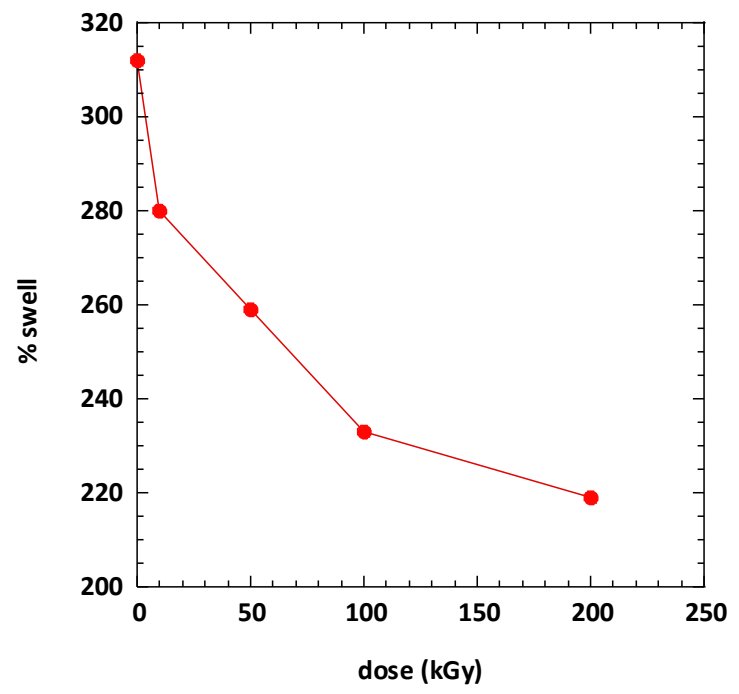

Figure 3. Percent swell in toluene of AM pads as a function of gamma dose. The swelling is limited by the cross-links formed as a result of radiolysis.

The irradiated AM pads were analyzed using FTIR spectroscopy as shown in Figure 4. The spectra are representative of silicone elastomers in general. A direct consequence of the removal of $\mathrm{H}$ atoms belonging to methyl groups would lead to a decrease in intensity of the band at ca. $2964 \mathrm{~cm}^{-1}$, which corresponds to the $\mathrm{C}-\mathrm{H}$ stretching in $\mathrm{CH}_{3}$. Another expected effect of radiolysis would be a decrease in intensity of the ca. 1261 and $799 \mathrm{~cm}^{-1}$ bands $\left(\mathrm{CH}_{3}\right.$ symmetrical bending and $\mathrm{CH}_{3}$ rocking, in $\left.\mathrm{Si}-\mathrm{CH}_{3}\right)$. Finally, an increase in cross-link density has been associated with changes in the broad band at ca. $1092 \mathrm{~cm}^{-1}$ and $1020 \mathrm{~cm}^{-1}$, which are related with asymmetric and symmetrical stretching of Si-O-Si; respectively. ${ }^{18}$ The FTIR spectra of irradiated AM pads show a decrease in the $\mathrm{CH}_{3}$ peaks at $1400 \mathrm{~cm}^{-1}, 1261 \mathrm{~cm}^{-1}$, and changes in the broad band at $1092 \mathrm{~cm}^{-1}$ as well as on the shoulder of the peak at $799 \mathrm{~cm}^{-1}$. These results 
indicate that small chemical changes occurred in the AM pad due to the ionizing exposure and are in agreement with cross-link formation.

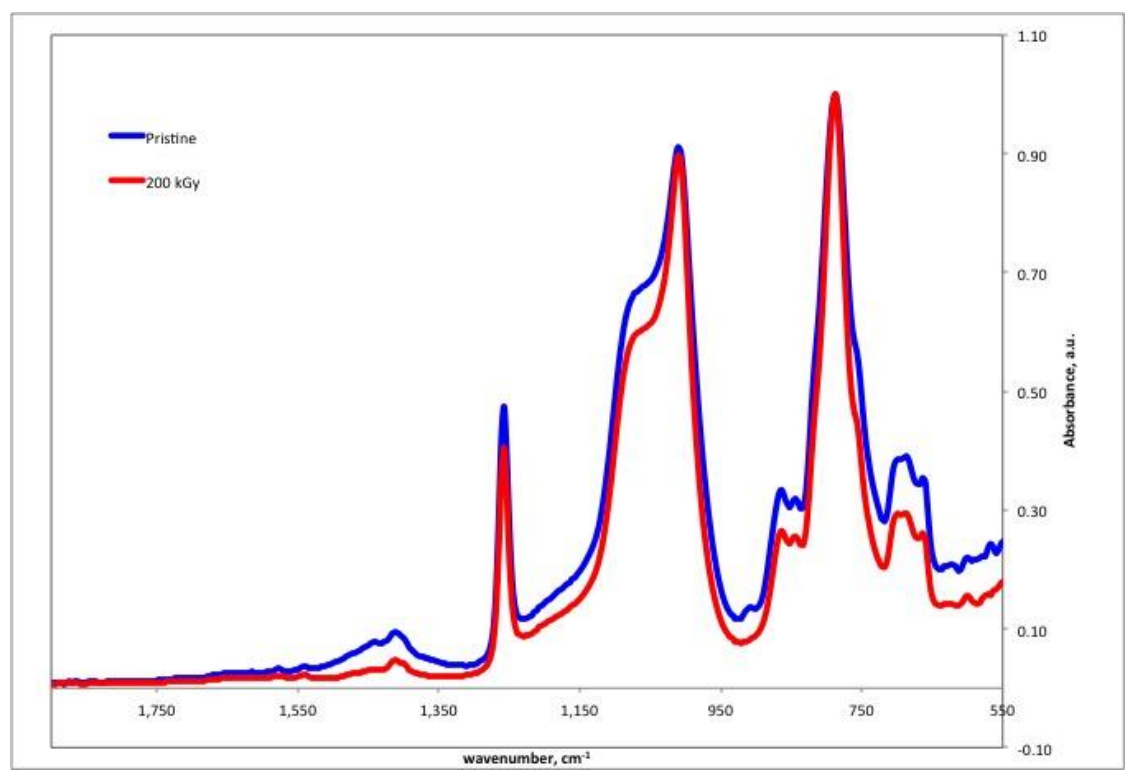

Figure 4. FT-IR absorbance spectra for AM pad exposed to 200kGy (red trace) and control sample (blue trace).

Changes in the chemistry of the irradiated AM pads were also investigated by NMR spectroscopy. Extracted material was obtained from the AM pad with the objective to isolate and detect fragments due to radiation damage like chain scission. Extracts were analyzed by liquid-state ${ }^{1} \mathrm{H}$ NMR spectroscopy and were found to be composed mainly of PDMS chains. Quantitative analysis of the extracts showed no significant changes in their concentration with radiation exposure. No fragments were detected that could be associated with chain scission in agreement with cross-link formation being the predominant effect of radiolysis.

The investigation of stable radical species was of interest to this study to better understand radiolysis effects in this commercial resin. EPR spectroscopy was thus performed to monitor the presence of any stable radical species formed by exposure to ionizing radiation. The irradiated AM pads were probed by X-band EPR spectroscopy at room temperature; the results are shown in Figure 5. Although there are broad features observed over the span of the 
spectra and some structure near $3000 \mathrm{G}$, it is challenging to discern any trends between the irradiated samples and the control sample. Even subtractions of the spectrum of an empty tube from the spectra of the irradiated samples show no correlation to irradiation dose. Thus, the radicals formed on irradiation are supposed to be unstable.
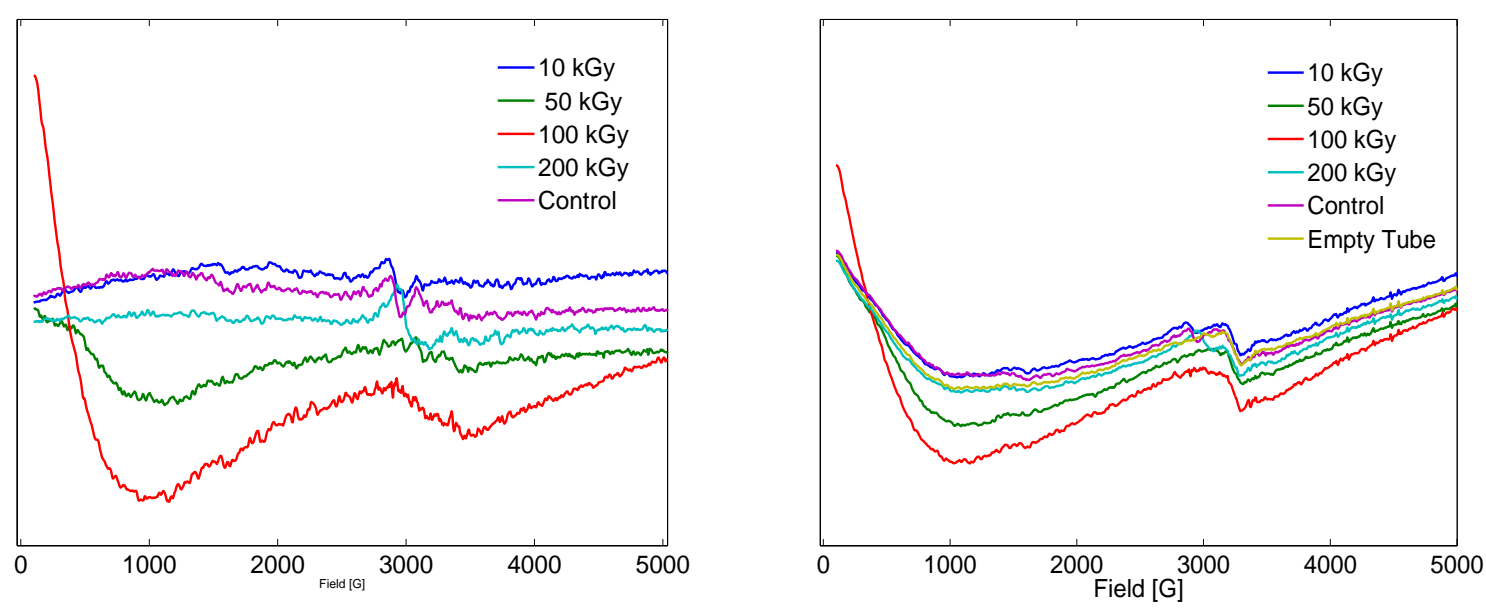

Figure 5. X-band CW EPR spectra (left) obtained of AM pads that were irradiated to doses of 10, 50, 100 and $200 \mathrm{kGy}$. (right) Subtraction of the irradiated samples spectra from the spectrum of an empty tube (background). Spectra were acquired at room temperature using a microwave power of $5 \mathrm{~mW}$.

\subsection{Irradiation Effects on Thermal Properties.}

It is well known that PDMS thermal degradation in an inert atmosphere and under vacuum results in depolymerization and evolution of cyclic oligomers, with the trimer being the most abundant product. ${ }^{19,20}$ The degradation rate and residual mass can vary over a large range (0 to 29\%) depending on factors such as impurities, water, fillers and residual catalyst. For instance, the incorporation of ppm-levels of cesium octoate to the polymer significantly increases the residual mass. ${ }^{21,22}$ TGA experiments were conducted to evaluate changes in the thermal stability of the irradiated SE1700 AM pads. The evolving weight $\%$ and the derivative weight $\%$ (DW\%) are shown in Figure 6. There are two distinct regions of mass loss, which were 
identified by studying peak locations in the DW\% data. For all samples, measurable mass loss starts at around $200^{\circ} \mathrm{C}$ and maxes out at $420^{\circ} \mathrm{C}$. The degradation rate was slightly slower for the samples exposed to the largest gamma doses, reflecting an enhancement of thermal stability with radiation. This result likely suggests an increasing in cross-linking density, which is expect to inhibit degradation by decreasing chain mobility. In contrast, the opposite behavior is observed above $638^{\circ} \mathrm{C}$ where TG curves crossed-over. The thermal decomposition rate becomes faster for the most irradiated samples when compared to the least exposed ones. Once thermal degradation ended, less residual mass was observed with increasing gamma dose. This trend is not likely due to sample variations, since the AM pads were from the same batch. A possible explanation for the observed trend could be the existence of free-radicals. However, EPR experiments found no evidence correlating free-radical concentration with gamma exposure. It is worth pointing out that our EPR experiments were performed at room temperature. Based on this reasoning, we infer that high temperatures promoted radical formation that resulted in faster degradation rates and lower residual masses. It seems that the abundance of these high-temperature species is dependent on the accumulative gamma dose.

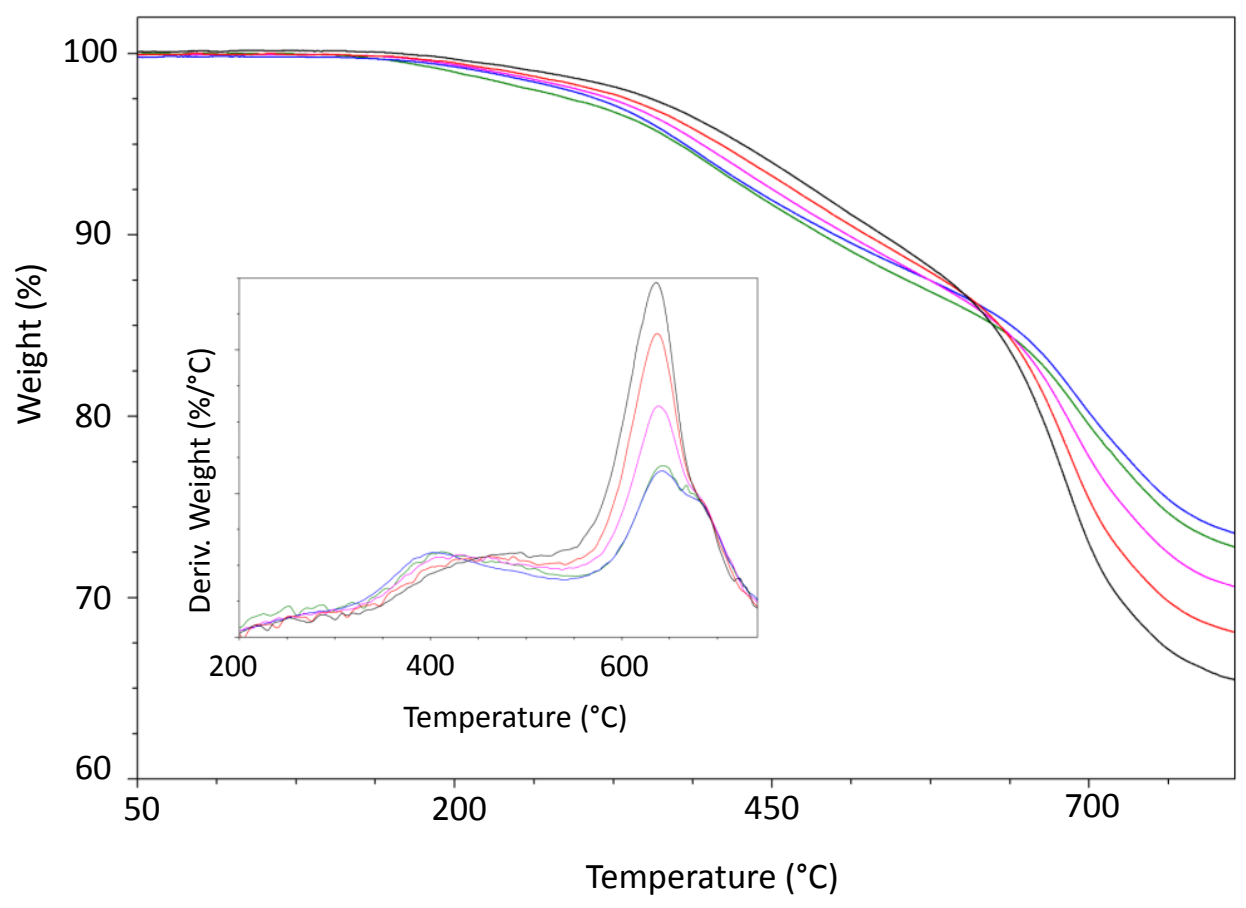


Figure 6. TGA results show AM pads undergoing mass loss during a constant ramp in temperature. The derivative weight $\left(\% /{ }^{\circ} \mathrm{C}\right)$ is given in the inserted figure. Key: control (green trace), $10 \mathrm{kGy}$ (blue trace), $50 \mathrm{kGy}$ (pink trace), $100 \mathrm{kGy}$ (red trace), and $200 \mathrm{kGy}$ (blue trace).

DSC was applied to investigate variations in crystallization phenomena and glass transition temperature $\left(T_{g}\right)$ as promoted by the ionizing radiation. DSC results are shown in Figure 7 . The thermograms exhibit a well-defined exothermic peak in the cooling cycle as well as an endotherm peak in the heating cycle. Additionally, $\mathrm{T}_{\mathrm{g}}$ can be observed at about $-120^{\circ} \mathrm{C}$. No noticeable temperature shift in $\mathrm{Tg}$ was detected as a function of gamma dose, but crystallization and melting temperatures shifted to lower temperatures and broaden considerable with increasing radiation exposure. The crystallization temperature decreased from $-71.6^{\circ} \mathrm{C}$ for the control sample to $-89.6^{\circ} \mathrm{C}$ for the $200 \mathrm{kGy}$ AM pad. Likewise, the melt temperature decreased from $-44.5^{\circ} \mathrm{C}$ for the control sample to $-50.8^{\circ} \mathrm{C}$ for the AM pad exposed to $200 \mathrm{kGy}$. Overall, a decrease in the estimated amount of crystallinity is observed with increased radiation dose. The initial crystallinity of the AM pad is close to $32 \%$, but it decreases to about $26 \%$ for the sample receiving the highest gamma exposure (\% crystallinity $=100 * \Delta \mathrm{H}_{\mathrm{f}} / \Delta \mathrm{H}_{\mathrm{f}}{ }^{0}$, where $\Delta H_{f}$ is the measured heat of fusion under the melting endotherm and $\Delta H_{f}{ }^{\circ}$ is the heat of fusion of the $100 \%$ crystalline polymer, with $\Delta \mathrm{H}_{\mathrm{f}}{ }^{0}=61.3$ or $37.4 \mathrm{~J} / \mathrm{g}$, we used $37.4 \mathrm{~J} / \mathrm{g}){ }^{23,24}$ In addition, the pads irradiated to 100 and $200 \mathrm{kGy}$ revealed a second broad peak at temperatures close to $\mathrm{T}_{\mathrm{g}}$, for both crystallization and melt events. These results are consistent with an increase in cross-link density, which essentially prevents chain conformational mobility and limits the reconfiguration of the chains into a crystalline state. Thus, cross-linking formation decreases the degree of crystallinity as well as the stability of the crystalline phase. ${ }^{23}$ Changes in crystallinity levels are expected to influence the low temperature mechanical response, such as modulus, yield stress, and creep. Mechanical tests at low temperatures were then conducted to assess radiolysis effects and are discussed later on in this work. 

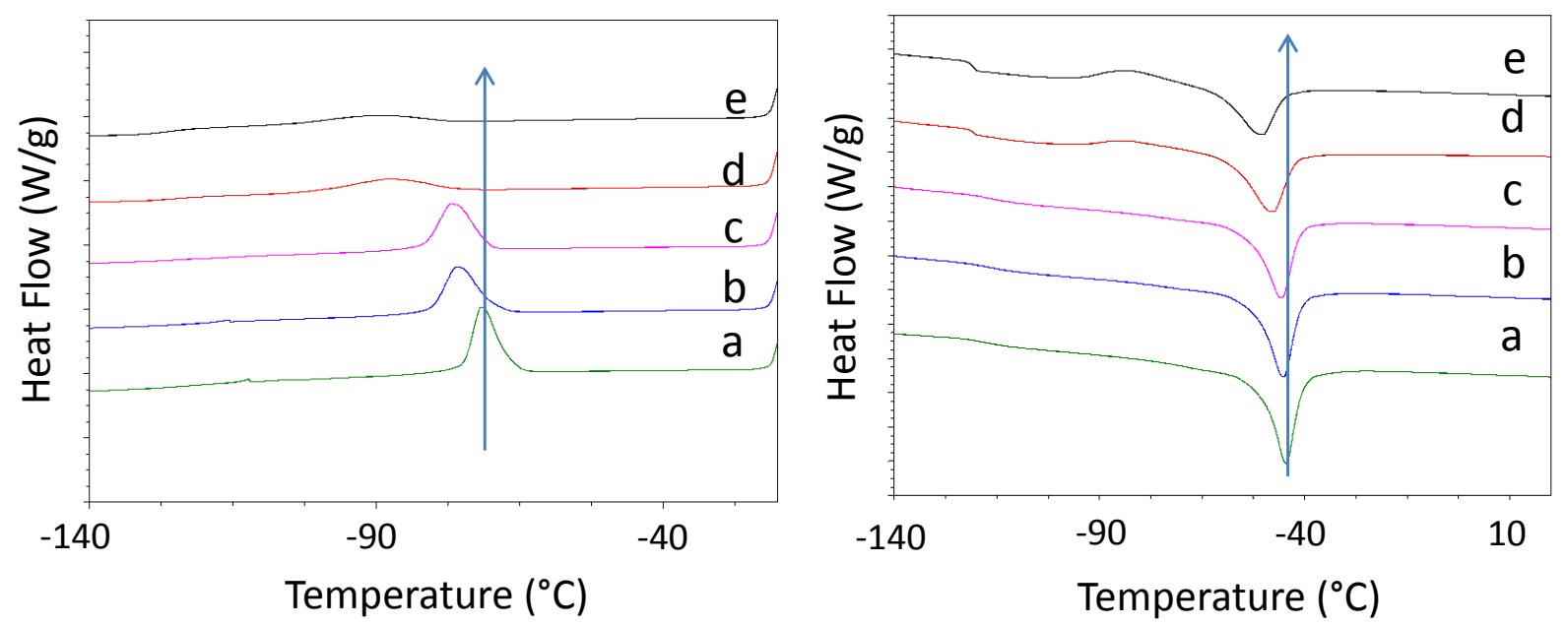

Figure 7. DSC thermograms of irradiated and control AM pads. These figures show the change in heatflow as a result of the gamma dose for cooling (left), and heating (right). The control sample (a), $10 \mathrm{kGy}$ (b), $50 \mathrm{kGY}$ (c), $100 \mathrm{kGy}(\mathrm{d})$, and $200 \mathrm{kGy}(\mathrm{e})$. (The arrows illustrate the shifts in temperature with gamma exposure).

\subsection{Irradiation Effects on Microstructure.}

Changes in the microstructure of the AM pad were investigated by SEM with relevant images shown in Figure 8. Our results indicate that samples subjected to the highest gamma doses exhibited micro-cracks on their surface when compared to the control sample. Less damage was observed for the samples exposed to lower doses, indicating that these were systematic changes that could unambiguously be attributed to the radiation exposure. A few studies published in the literature of irradiated polysiloxane foams indicate that changes in the microstructure are dependent on radiation conditions and chemistry of the polymer. For instance, Wei and co-workers found that the open and spherical microstructure of a siloxane foam collapsed after being exposed to 1 MGy dose (which is 5 times higher than our highest dose). ${ }^{25}$ On the other hand, our team investigated damages to the microstructure of a room temperature vulcanized foam when exposed to similar doses as the ones used here and found 
no evidence of damage. ${ }^{26}$ The RTV foam contained diphenyl groups, which are known to increase the material's tolerance to radiolysis. ${ }^{27}$

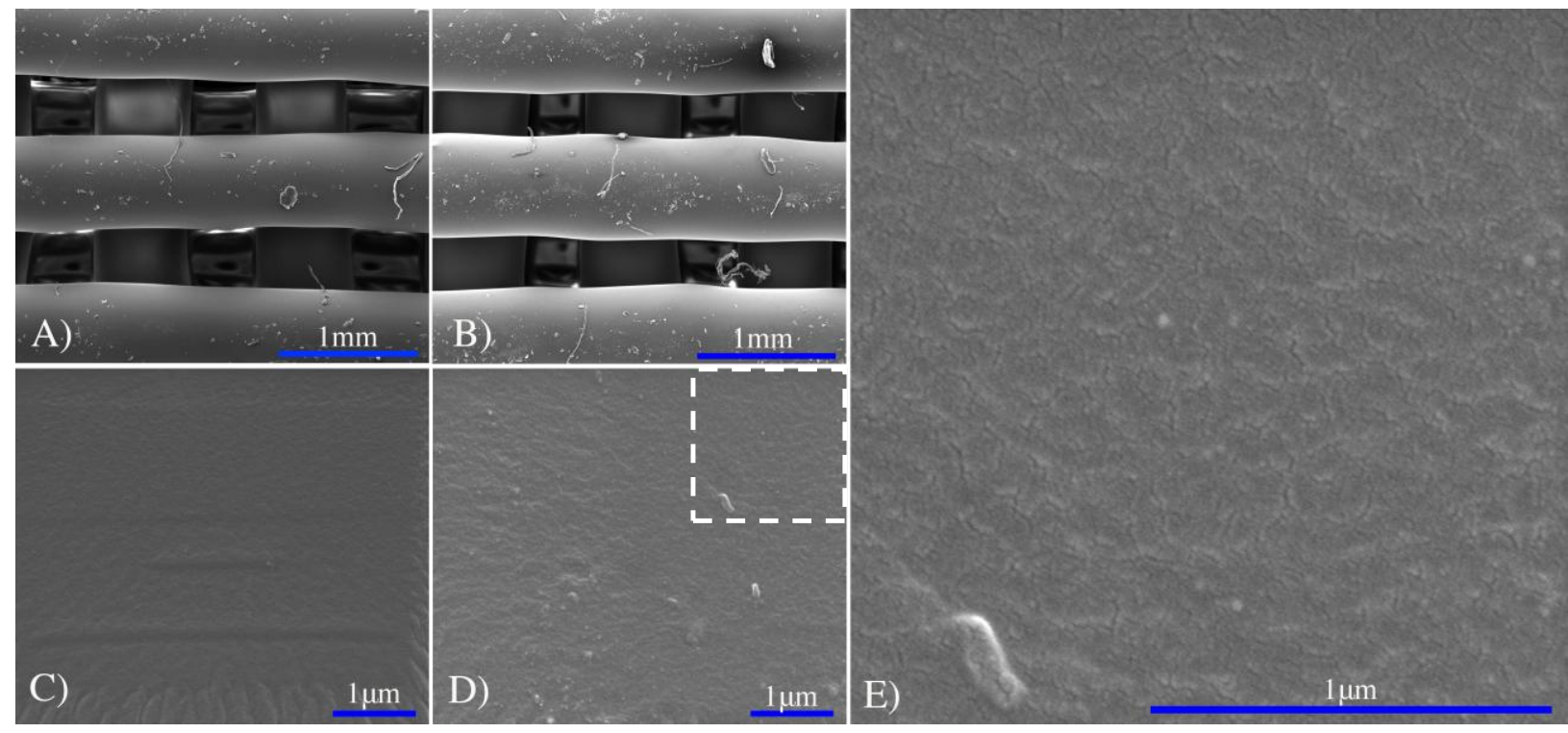

Figure 8. Large-scale images of the AM pad show the struts that make up the 3-D structure (A and B). The sample irradiated to the highest dose, $200 \mathrm{kGy}(\mathrm{B}, \mathrm{D}, \mathrm{\&} E)$ shows small surface cracks in the nanometer length-scale when compared to the control sample ( $A$ and $C$ ). A blown up image of the damaged sample detailing the micro-cracks is also shown in $E$, which represents the upper right hand corner of $D$ (given by the dashed white box).

\subsection{Irradiation Effects on Mechanical Properties.}

Mechanical tests were performed at variable temperature in order to better understand the material's response below and above crystallization. Tests were run at room temperature and at $-60^{\circ} \mathrm{C}$, which is near the temperature in which a crystalline phase transition is observed in DSC experiments. Results of cyclic loading at room temperature are shown in Figure 9. The elastic regime of the bulk material is well exceeded in our tests, as evidenced by the nonlinear shape of the stress-strain curve. This means that above $~ 50 \%$ strain the AM structure collapses, leaving the pad to respond similarly as the fully dense material. It is interesting to note that a 
non-reversible hysteresis is observed after the first loading cycle, which corresponds to the well-known Mullins effect. ${ }^{28,29}$ This stress softening of the pad is detected for all samples and is likely due to lessening of physical interactions between the surface of the silica filler and neighboring polymer chains. Furthermore, increasing in stiffness with gamma dose was also observed. This is in agreement with previous polymer durometry data showing increase in Shore A hardness ${ }^{9}$, which can be interpreted as an increase in the Young's modulus. ${ }^{30}$ The observed increase in stiffness is thus associated with radiation-induced cross-link of the polymer chains. In addition to cross-link formation, Maxwell and co-workers observed enhancement of polymer-filler interactions when similar materials were subjected to gamma radiation. ${ }^{31}$ Their reasoning was that additional active sites on the filler's surface are created due to ionizing radiation exposure. Mechanical tests performed here show some evidence to substantiate this notion, since the Mullins effect seems to be more pronounced for the irradiated pads than for the control one.

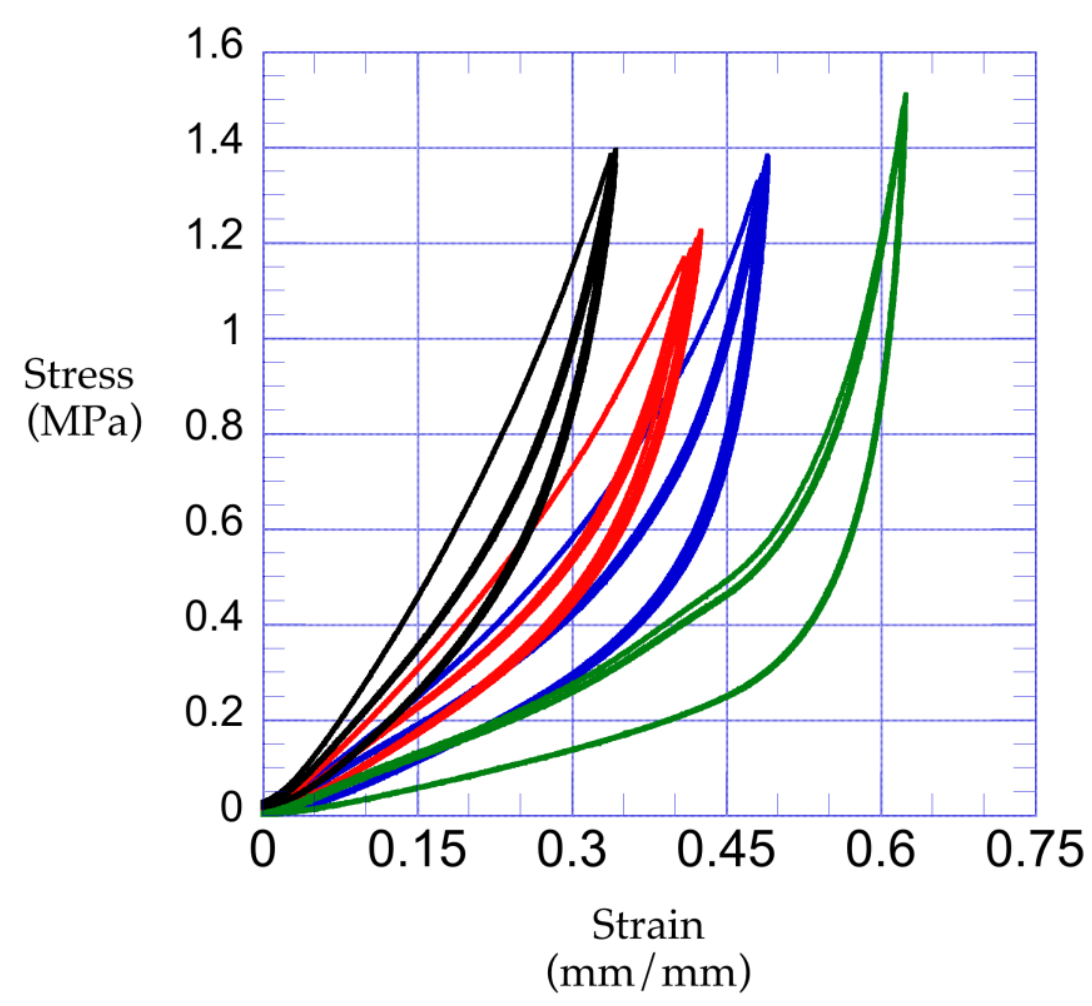

Figure 9. Engineering stress as a function of the engineering strain observed for the irradiated AM pads, control (green trace), 10 kGy (blue trace), 100 kGy (red trace), and 200 kGy (black 
trace) sample. The AM pads were compressed cycled four times at $20^{\circ} \mathrm{C}$ at a strain rate of $0.01 / \mathrm{s}$.

Figure 10 shows the low temperature stress-strain response of irradiated pads. As expect, the material stiffens at low temperatures when compared to its room temperature response. Furthermore, the effect of gamma exposure on the degree of crystallization is also noticed by these mechanical tests. At $-60^{\circ} \mathrm{C}$, the stiffness of the material did not increase as a function of radiation dose as observed at room temperature. Radiation-induced cross-links act to hinder the formation of crystallites, which function as physical cross-link sites. The low temperature mechanical response of irradiated pads is thus a combination of opposite effects. On one hand, decreasing in crystallization levels results in soften of the pad, whereas on the other hand, additional cross-links give rise to stiffening of the pad.
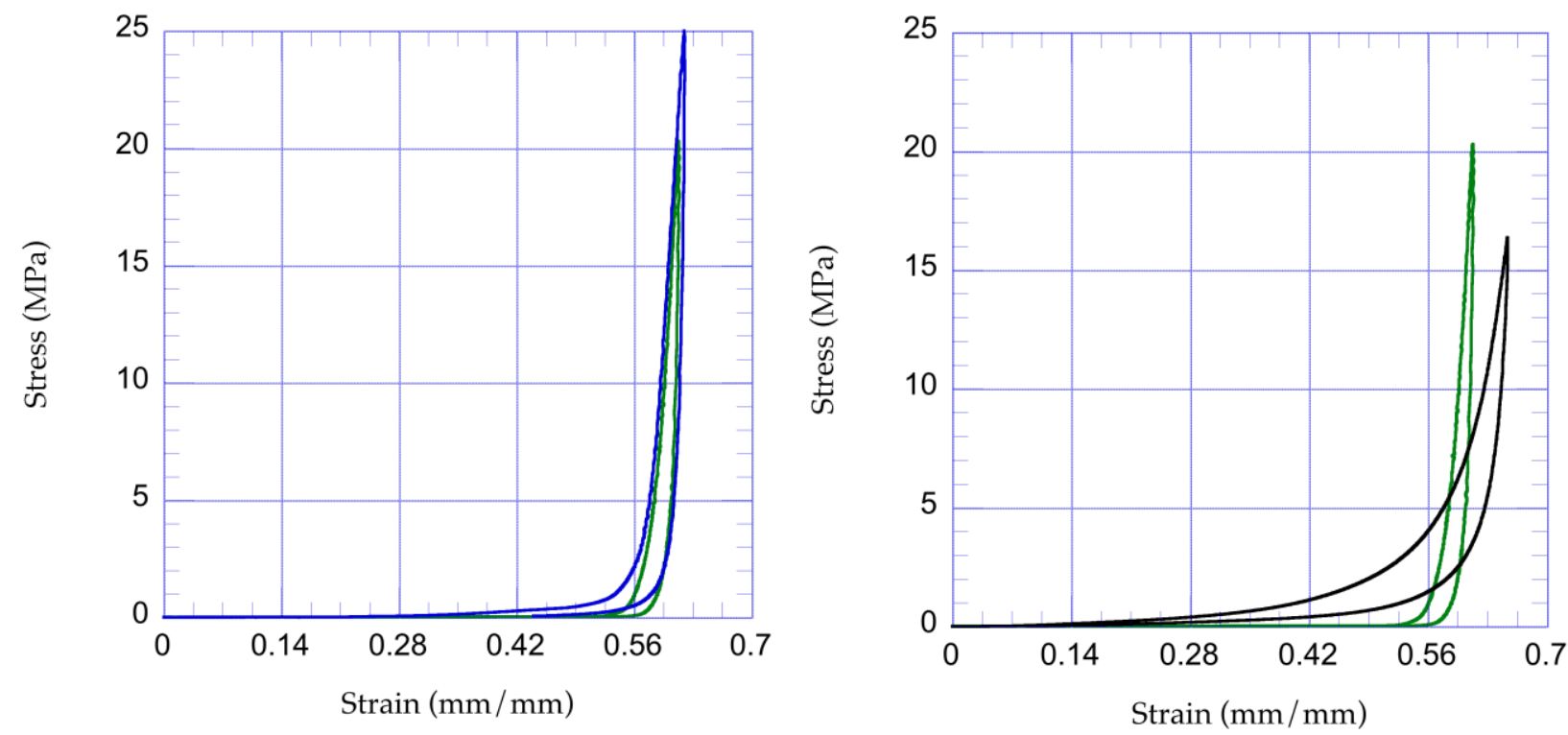

Figure 10. Engineering stress as a function of the engineering strain of AM pads obtained at $60{ }^{\circ} \mathrm{C}$. The plot on the left-side compares the stress-strain response of the $200 \mathrm{KGy}$ sample (blue) with the $100 \mathrm{KGy}$ (green) showing little stiffening due to a competition effect between increasing in cross-links and decreasing in crystallization. The plot on the right-side shows that at large strains the $100 \mathrm{KGy}$ sample (green trace) is slightly stiffer than the control one (black trace). 


\subsection{DWI resin containing phenyl groups.}

The lack of aromatic groups in the SE1700 resin implies that radiation effects become more important than if the resin contained a certain number of these species. According to studies performed by Delides, aromatic substitution in the polymer backbone provides remarkable protection against radiolysis; tolerance to ionizing radiation increases almost exponentially with increasing phenyl concentration. ${ }^{27}$ As an example of the protective effect provided by phenyl groups against radiation damage, it is interesting to compare our present results with those obtained by our team for a siloxane foam. ${ }^{26}$ In that study, a room temperature vulcanized (RTV) polysiloxane foam containing about 5wt\% diphenyl groups was irradiated under the same conditions as the present AM pads. Gas analysis of the evolved volatiles showed that the irradiated RTV foam produced, per gram of polymer, almost 2.5 times less hydrogen than the SE1700 AM pad.

As demonstrated in this work, AM pads manufactured using the SE1700 resin exhibit low resistance to radiation damage. In order to overcome this drawback, another commercially available Dow Corning ${ }^{\circledR}$ resin was modified in order to become printable. Fumed silica was added to the uncured material, turning it from a flowing resin to a yield stress one. This resin, identified as OE6636, has high phenyl content which is expect also to improve thermal stability of the polymer. For instance, the onset degradation temperature of PDMS increases dramatically when methyl-phenyl siloxane or diphenyl siloxane are incorporated as copolymers. $^{32-34}$ The high phenyl content of OE6636 was confirmed by DSC measurements, which showed the glass transition temperature to shift to $0^{\circ} \mathrm{C}$. It has been shown that for random copolymers, $\mathrm{Tg}$ is related to the mole percent of diphenyl groups $(\mathrm{x})$ by the following relationship: $T_{g}=1.95 x-123$. Accordingly, the diphenyl mole fraction in OE6636 is very high or about $63 \%$. AM pads manufactured from this modified resin were subjected to $200 \mathrm{kGy}$ gamma dose and were compared to the corresponding SE1700 pads. Similarly to irradiated SE1700 pads, analysis of the headspace indicated evolution of permanent gases. But as shown in Table 3 , their concentrations were much lower. These results indicate that commercially available 
resins that exhibit attractive properties like radiation resistance may be appropriated modified to be used in DIW applications.

Table 3. Gas evolutions from irradiated AM pads made from SE1700 and modified OE6636 resins. Gas concentrations are reported in ppm/g of cured resin. Both pads were exposed to $200 \mathrm{kGy}$ at room temperature under $\mathrm{N}_{2}$ and at same dose rate.

\begin{tabular}{|c|c|c|c|c|}
\hline AM resin & $\mathrm{H}_{2}$ & $\mathrm{CH}_{4}$ & $\mathrm{C}_{2} \mathrm{H}_{6}$ & $\mathrm{C}_{6} \mathrm{H}_{6}$ \\
\hline SE1700 & 3,332 & 2,939 & 373 & none \\
\hline OE6636 & 280 & 91 & 90 & 168 \\
\hline
\end{tabular}

\section{Conclusions.}

This work investigated the radiation tolerance of AM pads produced by the DIW technique. 3-D structures were printed using commercial PDMS-based resins. Our results demonstrated that an aromatic-free siloxane resin, known as SE1700, experienced chemical changes as evidenced by analysis of the evolved gases, which were predominantly hydrogen, methane and ethane. These results, coupled together with decreasing in solvent swelling, indicated that exposure to gamma irradiation induced formation of cross-links. The change of chemistry caused significant modifications to the thermal stability, microstructure and mechanical properties of the AM pad as well. TGA experiments showed that the onset of the thermal decomposition occurred at higher temperatures with increasing gamma exposure, whereas less crystallization and lower melting temperature were evidenced by DSC results. SEM experiments also detected the presence of nano-cracks on the surface of the irradiated pad. Compressive loading tests showed increasing in the stiffness response to controlled strains with increasing gamma dose. On the other hand, inhibition of crystallization by newly formed crosslinks produced less stiffened materials at low temperatures. A commercially available PDMSbased resin containing high amounts of diphenyl groups was also evaluated. Addition of silica to 
this resin allowed for AM pads to be 3-D printed. As expected, the modified material showed good resistance to radiolysis as evidenced by the low concentration of evolved gases in the headspace.

Acknowledgements. Tom Robinson and Stephanie Schulze from the National Security Campus in Kansas City, MO are thanked for providing the SE1700 AM pads. Don Hanson and Maryla Wasiolek from Sandia National Laboratory in Albuquerque, NM are thanked for their help with exposing the samples to gamma irradiation. We thank the Dynamic Materials Properties and the Enhanced Surveillance campaigns for proving financial support, under the US Department of Energy's National Nuclear Security Administration contract DE-AC52-06NA25396. 


\section{References.}

(1) Gibson, I.; Rosen, D. W.; Stucker, B.; Springer: New York, 2010.

(2) Toohey, K. S.; Sottos, N. R.; Lewis, J. A.; Moore, J. S.; White, S. R. Nat Mater 2007, 6, 581 .

(3) Durmus, N. G.; Tasoglu, S.; Demirci, U. Nat Mater 2013, 12, 478.

(4) Duoss, E. B.; Weisgraber, T. H.; Hearon, K.; Zhu, C.; Small, W.; Metz, T. R.; Vericella, J. J.; Barth, H. D.; Kuntz, J. D.; Maxwell, R. S.; Spadaccini, C. M.; Wilson, T. S. Adv Funct Mater 2014, 24, 4905.

(5) Charlesby, A. Proceedings of the Royal Society of London Series aMathematical and Physical Sciences 1955, 230, 120.

(6) Stpierre, L. E.; Dewhurst, H. A.; Bueche, A. M. J Polym Sci 1959, 36, 105.

(7) Miller, A. A. J Am Chem Soc 1960, 82, 3519.

(8) Warrick, E. L. Ind Eng Chem 1955, 47, 2388.

(9) Basfar, A. A. Radiation Physics and Chemistry 1997, 50, 607.

(10) Charlesby, A. J Polym Sci 1955, 17, 379.

(11) Belova, V. V.; Pridachina, N. N.; Popova, A. I.; Serenkov, V. I. RADIOLYSIS OF POLYSILOXANE RESIN, 1971.

(12) Bueche, A. M. J Polym Sci 1956, 19, 297.

(13) Hill, D. J. T.; Preston, C. M. L.; Whittaker, A. K.; Hunt, S. M. Macromol Symp 2000, 156, 95.

(14) Hill, D. J. T.; Preston, C. M. L.; Salisbury, D. J.; Whittaker, A. K. Radiation Physics and Chemistry 2001, 62, 11.

(15) Hill, D. J. T.; Preston, C. M. L.; Whittaker, A. K. Polymer 2002, 43, 1051.

(16) Labouriau, A.; Cady, C.; Gill, J.; Stull, J.; Ortiz-Acosta, D.; Henderson, K.; Hartung, V.; Quintana, A.; Celina, M. Polymer Degradation and Stability 2015, 116, 62. 854.

(17) Lee, S.; Pawlowski, H.; Coran, A. Y. Rubber Chemistry and Technology 1994, 67,

(18) Laboratories, S. R. The Infrared spectra atlas of monomers and polymers; Sadtler Research Laboratories, 1980.

(19) Thomas, T. H.; Kendrick, T. C. J Polym Sci A2 1969, 7, 537.

(20) Lewis, C. W. J Polym Sci 1959, 37, 425.

(21) Lin, S. B. In Acs Sym Ser; American Chemical Society: 1995; Vol. 603, p 37.

(22) Deshpande, G.; Rezac, M. E. Polymer Degradation and Stability 2002, 76, 17.

(23) Roland, C. M.; Aronson, C. A. Polym Bull 2000, 45, 439.

(24) Aranguren, M. I. Polymer 1998, 39, 4897.

(25) Wei, H.; Fu, Y. B.; Wang, C. Y.; Xu, Y. S.; Bian, Z. S. Radiation Physics and Chemistry 2002, 64, 229.

(26) Labouriau, A.; Cady, C.; Gill, J.; Taylor, D.; Zocco, A.; Stull, J.; Henderson, K.;

Wrobleski, D. Polym Degrad Stabil 2015, 117, 75.

(27) Delides, C. G. Radiation Physics and Chemistry 1980, 16, 345.

(28) Mullins, L. Rubber Chemistry and Technology 1969, 42, 339.

(29) Diani, J.; Fayolle, B.; Gilormini, P. European Polymer Journal 2009, 45, 601.

(30) Mix, A. W.; Giacomin, A. J. J Test Eval 2011, 39, 696.

(31) Maxwell, R. S.; Cohenour, R.; Sung, W.; Solyom, D.; Patel, M. Polymer Degradation and Stability 2003, 80, 443. 
(32) Grassie, N.; Macfarlane, I. G. Eur Polym J 1978, 14, 875.

(33) Grassie, N.; Francey, K. F. Polym Degrad Stabil 1980, 2, 53.

(34) Grassie, N.; Francey, K. F.; Macfarlane, I. G. Polym Degrad Stabil 1980, 2, 67. 